\title{
Heart Rate Variability Assessment using the Poincare Plot Parameters during Sleep
}

\author{
Jae Mok Ahn ${ }^{1}$ \\ ${ }^{1}$ Professor, School of Software, Hallym University, Chuncheon, Gangwon-do, South Korea.
}

\begin{abstract}
A nonlinear Poincare plot method for heart rate variability (HRV) analysis is widely used to evaluate autonomic nervous system activity. However, during sleep, the Poincare plot parameters are not described completely because an ectopic heartbeat, which is similar to an artifact, depends largely on the result of the Poincare plot. Therefore, in this study, we investigated the characteristics of four Poincare plot parameters to differentiate between rapid eye movement (REM) and nonrapid eye movement (NREM) after the removal of any artifacts. For the sleep Poincare plot analysis, two 10 min target HRV segments were obtained for each of the REM and NREM sleep stages from 16 individual subjects without autonomic disease. With a 2 min moving window with a time shift of $2 \mathrm{~s}, 240$ results were created and led to the formation of cloud points (or ellipses) on the Cartesian coordinate system. The length (SD1) and width (SD2) of an ellipse, ellipse area (Ln sArea), and $\mathrm{SD} 2 / \mathrm{SD} 1$ ratio were calculated to obtain the mean value and standard deviation (SD) of all the results. The SD2/SD1 ratio and Ln sArea exhibited excellent discrimination ability between REM and NREM in terms of their mean and SD ( $\mathrm{p}=$ 0.0001 and $p=0.0003$, respectively; $95 \% \mathrm{CI}$ ), but SD1 did not ( $p=0.9606$ for mean and $p=0.0320$ for SD). The Ln sArea remained nearly constant and was independent of the sleep stages. The results indicate that the Ln sArea and SD2/SD1 ratio could be used to assess physiological changes and health status during sleep and to discriminate between REM and NREM, respectively.
\end{abstract}

Keywords: Heart rate variability, rapid eye movement, Poincare plot, autonomic nervous system, sleep.

\section{INTRODUCTION}

Heart rate variability (HRV) analysis is of interest to clinicians and biomedical engineers. HRV provides useful information that can quantify the activity of the autonomic nervous system (ANS) by means of power spectral estimates and nonlinear estimates, which consist of the parasympathetic nervous system (PNS) and sympathetic nervous system (SNS) [1-4]. A nonlinear diagnostic parameter that can be obtained through a Poincare plot analysis is the result of a graphical representation of the difference between consecutive normal-to-normal (NN) heartbeat intervals in a time series in milliseconds [5-8]. This graphical expression includes the shape, size, dispersion, position, clusters, and localization for all the HRV data points on the Cartesian coordinate system. The mental, physical, psychological, and physiological states of a person are related to these graphical parameters owing to changes in body conditions, which are caused by external and internal stimuli [2]. Therefore, recently, nonlinear HRV analysis, such as the Poincare plot, has been increasingly applied in various clinical applications [9-11]. However, during sleep, specifically the Poincare plot analysis is not understood as much as other nonlinear estimates, such as entropy-derived parameters or other linear fast Fourier transform estimates, which have very low frequency, low frequency (Ln LF), and high frequency (Ln $\mathrm{HF}$ ) in the frequency domain. Therefore, this study aimed to investigate the characteristics of nonlinear Poincare plot parameters to differentiate between rapid eye movement (REM) and non-rapid eye movement (NREM) during sleep. From the perspective of ANS operations, sleep is characterized by a dynamic change in both the PNS and the SNS, with a mix of many biological systems, such as neural, physiological, and cardiac autonomic activities [12-14]. Most sleep studies using the linear HRV methodology include an evaluation of the discrimination performance between REM and NREM, and the transition from REM to NREM or vice versa, and between sleep stages, but few include sleep interpretation using nonlinear HRV methodology such as the Poincare plot [15]. Therefore, to perform the sleep Poincare analysis of the HRV dataset, a pair of two 10 min target HRV datasets for each of the REM and NREM sleep stages for 16 individual subjects was prepared. A 10 min HRV segment was analysed by shifting a predetermined time length, i.e., a 2 min window with a time shift of $2 \mathrm{~s}$, to obtain 240 results of each Poincare plot parameter to calculate their average and standard deviation (SD). There was a statistical difference between REM and NREM in terms of the mean and SD $(p=0.0001$ and $p=0.0003$; 95\% CI) for the SD2/SD1 ratio and Ln sArea, respectively. Meanwhile, there was no significant difference between REM and NREM ( $p=0.9606$ and $p=0.0790 ; 95 \% \mathrm{CI})$ for the mean of SD1 and the Ln sArea, respectively. The results indicate that among nonlinear Poincare plot parameters, the SD2/SD1 ratio could be a useful predictor for differentiating between REM and NREM. 


\section{METHOD}

\section{II.I SUBJECTS}

Our study included 16 patients in good health (all males, age $(41.13 \pm 14.39$ years $)$, height $(169.87 \pm 9.74 \mathrm{~cm})$, weight $(73.60 \pm 13.80 \mathrm{~kg})$, BMI $(25.37 \pm 3.51)$, heart rate $(\mathrm{REM})$ $(66.33 \pm 6.63 \mathrm{bpm})$, and heart rate (NREM) $(65.33 \pm 7.67 \mathrm{bpm})$. Overnight polysomnography (PSG) (Nox-A1, Nox Medical Inc. Reykjavik, Iceland) was performed. Electrocardiography signals of the PSG were measured at a sampling frequency of $200 \mathrm{~Hz}$ during sleep. None of the subjects had a history of any medical condition to influence ANS function, such as obstructive sleep apnea, cardiac arrhythmias, and symptoms of sleep deprivation.

\section{II.II PROCESSING SCHEME}

The Poincare plot was analyzed by moving a 2 min window forward every $2 \mathrm{~s}$ for a 10 min target HRV segment, as shown in Fig. 1. The sleep Poincare plot parameters were calculated in the research mode of a pulse analyzer, TAS9VIEW (CANOPY9 RSA, IEMBIO Co., Ltd, Chuncheon-si, Republic of Korea) that we developed. Fig. 1 shows that, based on the overlapping segments, a 2 min window slides forward to calculate 240 results for each target HRV segment, all of which are used to obtain the mean and SD.

\section{II.III POINCARE PLOT ANALYSIS}

Poincare plots based on the nonlinear graphical analysis of NN time series provide clinical information, such as on sleep disorders, cardiovascular symptoms, and physiological changes [16]. To draw the nonlinear Poincare HRV graph, each heartbeat $\mathrm{NN}[\mathrm{n}-1]$ was plotted against the next heartbeat $\mathrm{NN}[\mathrm{n}]$. Each pair of the previous $\mathrm{NN}[\mathrm{n}-1]\left(\mathrm{x}_{\mathrm{p}}\right)$ and next $\mathrm{NN}[\mathrm{n}]\left(\mathrm{x}_{\mathrm{n}}\right)$ intervals has a Cartesian coordinate system, $\left(x_{p}, x_{n}\right)$, where $x_{p}$ is the previous heartbeat, and $x_{n}$ is the next heartbeat. For example, if the heartbeat vector consists of $\mathrm{NN}[\mathrm{n}]=(\mathrm{x}[0]$, $x[1], \ldots, x[n]$ ) with a data size $n=100$, the Poincare plot, which is known as a return map, will make a cloud of the points with coordinates $(\mathrm{x}[0], \mathrm{x}[1]),(\mathrm{x}[1], \mathrm{x}[2]),(\mathrm{x}[2], \mathrm{x}[3]), \ldots,(\mathrm{x}[99]$, $\mathrm{x}$ [100]). This return map forms a cloud of points at the center of a two-dimensional plane and locates the cloud on the line of identity. Whether the cloud points are above or below the line of identity determines the variability or fluctuations of the NN intervals in a time series. Therefore, the Poincare plot parameters can extract the ANS functioning from the changes in the distance between successive $\mathrm{NN}$ intervals, that is, the dispersion, thereby resulting in the formation of an ellipse with a longitudinal and transverse axis. To define the Poincare plot parameters mathematically, the ellipse fitting equation was used to calculate the SD of all the distances of the points perpendicular to the axis of the line of identity $(y=-x)$ and the $\mathrm{SD}$ of all the distances of the points along the axis of the line of identity $(\mathrm{y}=\mathrm{x})$, which were defined as SD1 (ellipse width) and SD2 (ellipse length), respectively [17]. Fig. 2 shows a graphical representation of SD1 and SD2 plotted to distinguish REM and NREM. The Poincare plot parameters are mathematically defined according to the following equations. Two auxiliary $\mathrm{NN}$ vectors defined in Equations (1) and (2) were created, and four Poincare plot parameters were determined in our previous study [18]:

$$
\begin{aligned}
& x^{a}=\left(x_{0}, x_{1}, \ldots, x_{N-1}\right) \\
& x^{b}=\left(x_{1}, x_{2}, \ldots, x_{N}\right) \\
& x^{c}=\frac{x^{a}-x^{b}}{\sqrt{2}}, \quad x^{d}=\frac{x^{a}+x^{b}}{\sqrt{2}}
\end{aligned}
$$

$$
\mathrm{SD} 1=\sqrt{\operatorname{variance}\left(x^{c}\right)}, \mathrm{SD} 2=\sqrt{\text { variance }\left(x^{d}\right)}
$$

$$
\begin{aligned}
& \mathrm{SD} 2 / \mathrm{SD} 1 \text { ratio }=\frac{S D 2}{S D 1} \\
& \mathrm{Ln} \text { sArea }=\log (\pi * \mathrm{SD} 1 * \mathrm{SD} 2)
\end{aligned}
$$

These Poincare plot parameters for the HRV datasets are characterized by the shape, size, and position of the cloud, localization, separation of the cloud, and symmetry of the data points on the Cartesian coordinate system [19].

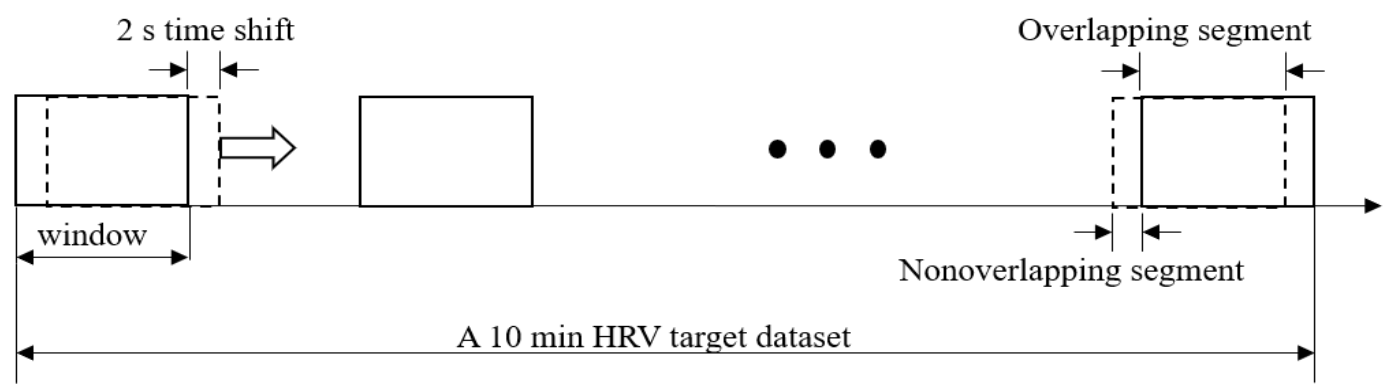

Fig. 1. Processing scheme for calculating Poincare plot parameters with 240 results after moving a 2 min window forward by $2 \mathrm{~s}$ for a 10 min target HRV dataset. 

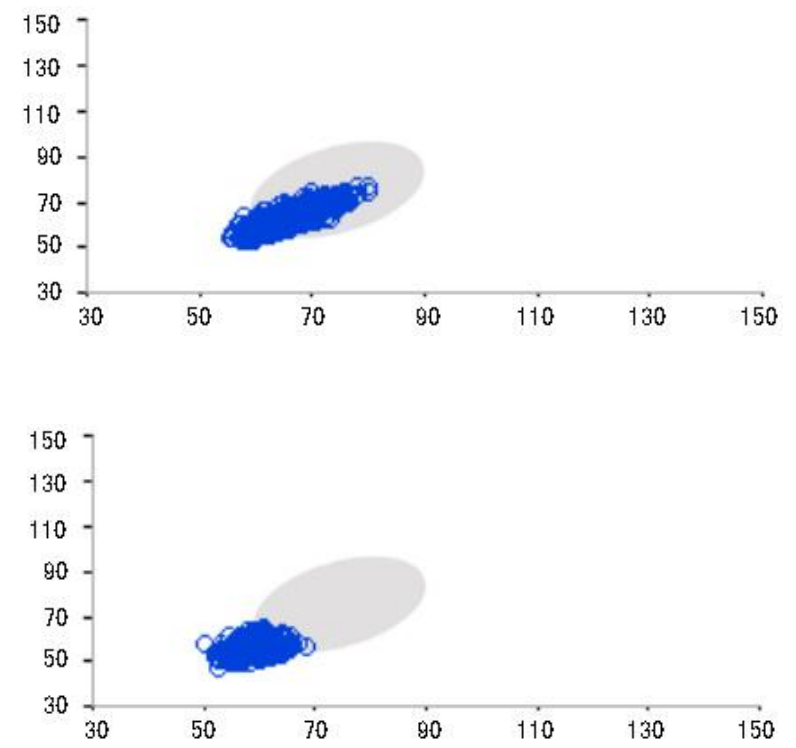

Fig. 2. Poincare plot of two different HRV datasets from (top) REM and (bottom) NREM.

\section{RESULT AND DISCUSSION}

Poincare plot parameters were investigated to differentiate REM and NREM in sleep stages by means of a 2 min moving window with a time shift of $2 \mathrm{~s}$ for a $10 \mathrm{~min}$ target HRV dataset. The mean of SD1 could not distinguish REM from NREM, but that of SD2 showed a statistically significant difference between REM and NREM ( $p=0.9606$ and $p=0.0004$, respectively; 95\% CI). The value of SD2 in the NREM sleep stage was found to decrease by half compared to that of SD2 in REM, as listed in Table 1. This observation is obvious evidence of parasympathetic predominance activation, which led to a diminished Ln LF/Ln HF ratio in a previous study [20]. An increase in SD2 relative to SD1 demonstrates a reduction in sympathetic activity, whereas the opposite demonstrates a reduction in parasympathetic activity. We found that the entropy-derived parameter, sample entropy (SampEn), increased more in NREM than in REM [20], indicating the high complexity of the HRV dataset. The higher the complexity of the HRV, the higher the decrease in SD2, and the higher the increase in parasympathetic activity over sympathetic activity. The most statistically significant difference was observed for the SD2/SD1 ratio ( $p=0.0001)$. This result suggests that there is high possibility that the SD2/SD1 ratio could be used as a meaningful predictor to distinguish REM from NREM. For the logarithmic value of the product of SD1 and SD2, Ln sArea, there was no significant difference between two sleep stages ( $p$ $=0.079)$. For the SD that refers to the fluctuations between insubject parameters, there were statistically significant differences between REM and NREM for SD2, the SD2/SD1 ratio, and Ln sArea, except for SD1 ( $p=0.0008, p=0.0016, p$ $=0.0003$, and $p=0.0320$, respectively). The fluctuation in the $\mathrm{SD}$ of the Ln sArea was larger in NREM than in REM (7.73 and $1.15 \mathrm{~ms}^{2}$, respectively).
Table 1. Paired t-test statistics to differentiate REM and NREM.

\begin{tabular}{ccccccc}
\hline \multirow{2}{*}{$\mathrm{n}=16$} & \multicolumn{2}{c}{ REM } & \multicolumn{2}{c}{ NREM } & \multicolumn{2}{c}{$p$-value } \\
\cline { 2 - 7 } & Mean & SD & Mean & SD & Mean & SD \\
\hline SD1 & 24.52 & 21.38 & 24.25 & 12.12 & 0.9606 & 0.0320 \\
SD2 & 75.60 & 35.73 & 36.56 & 13.31 & 0.0004 & 0.0008 \\
SD2/SD1 & 4.06 & 1.98 & 1.66 & 0.41 & 0.0001 & 0.0016 \\
Ln sArea & 8.25 & 1.15 & 7.71 & 7.73 & 0.079 & 0.0003 \\
\hline
\end{tabular}

Fig. 3 shows how much the mean of individual Poincare plot parameters differ between REM and NREM. SD1 is characterized by the least fluctuations between REM and NREM, where SD1 corresponds to Ln LF in the frequency domain. A much lower value of SD1 for both REM and NREM than SD2 was observed, resulting in a less dynamic change in sympathetic tone. The SD2/SD1 ratio may be used to assess the dynamics of HRV because its mean value is influenced most between REM and NREM.
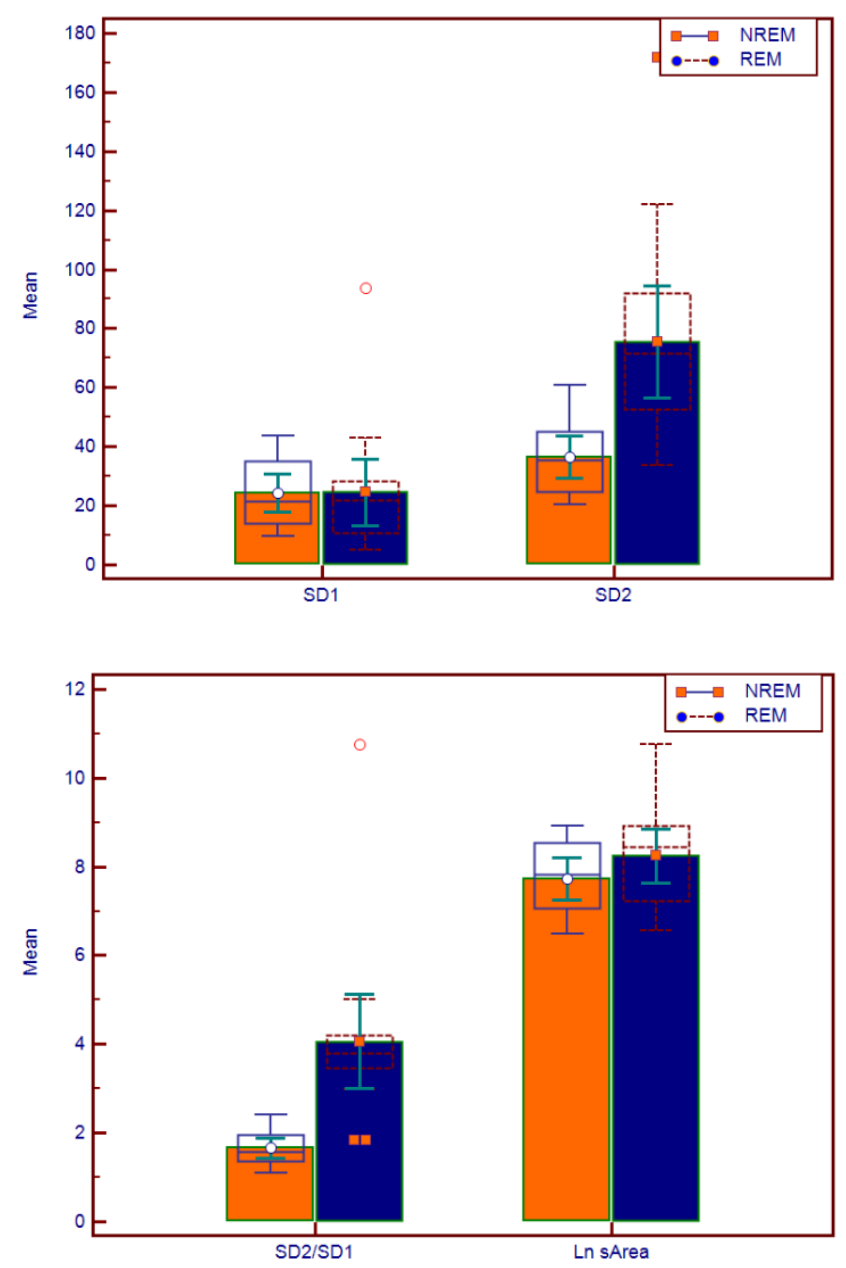

Fig. 3. Mean values of SD2/SD1 and Ln sArea for REM and NREM according to SD1 and SD2. 
We investigated how much the Poincare plot parameter fluctuates in sleep stages in terms of the SD as well, as shown in Fig. 4. The Ln sArea, which is from an ellipse with a longitudinal (SD2) and a transverse (SD1) axis, was found to have the least fluctuation, followed by SD1, SD2, and the $\mathrm{SD} 2 / \mathrm{SD} 1$ ratio. The low SD2/SD1 ratio suggests that there is a low correlation between $\mathrm{NN}$ intervals in a time series, while a high ratio indicates a high correlation between $\mathrm{NN}$ intervals, which reflects decreased randomness or regularity of HRV.
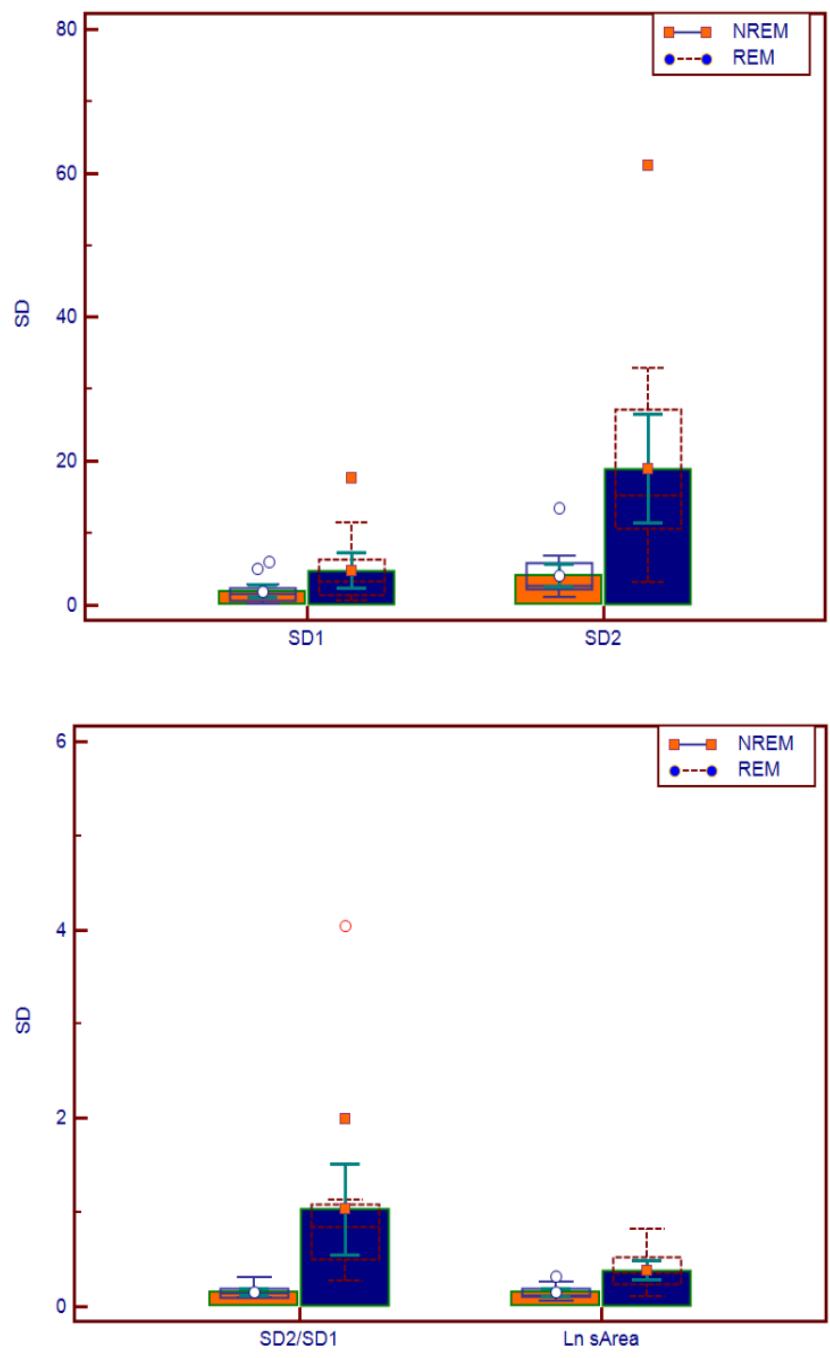

Fig. 4. SD between REM and NREM according to the SD2/SD1 ratio and Ln sArea, the area of an ellipse.

Fig. 5 shows how much each Poincare plot parameter changes in NREM with respect to REM. The proportion of NREM/REM (\%) was largest for SD1, although the average SD1 of the 16 subjects was not different between NREM and REM. All but one Poincare plot parameter, SD1, was reduced more in NREM. However, SD1, SD2, and the SD2/SD1 ratios were significantly altered in subjects during sleep compared to the area of an ellipse, Ln sArea, whose value remained relatively consistent. This result suggests that changing the NN interval dynamics leads to significant changes in both SD1 and SD2 between REM and NREM. The fact that the SD2/SD1 ratio between REM and NREM remains constant indicates that dynamic ANS activity is well reflected because a decrease in both SD1 and SD2 parameters during sleep results in a reduction in Ln sArea. It was reported that the SD2/SD1 ratio was significantly decreased in healthy subjects $(p=0.05)$ [19].

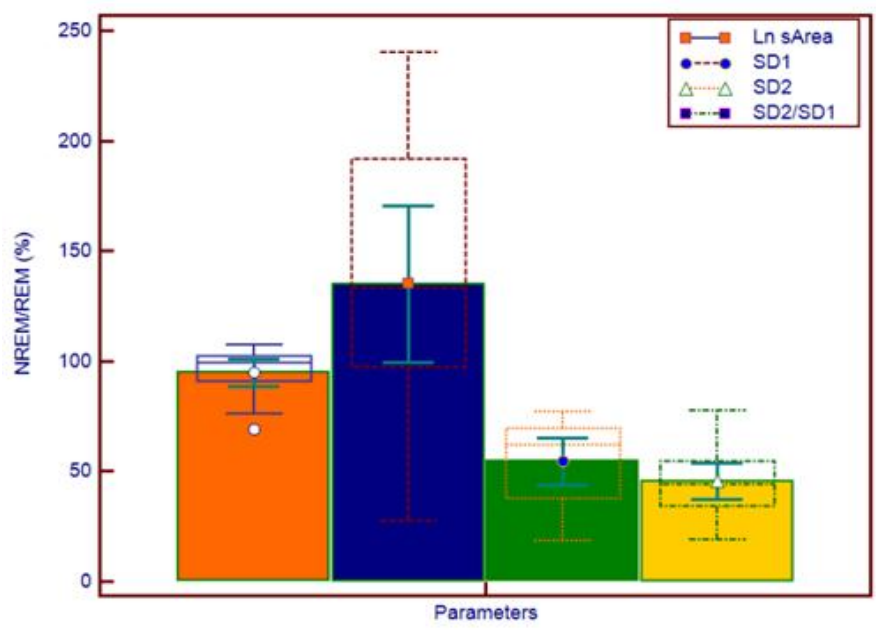

Fig. 5. Influence of Poincare plot parameters on REM and NREM.

All the profiles of the 240 results for SD1, SD2, the SD2/SD1 ratio, and $\mathrm{Ln}$ sArea were plotted to evaluate the discrimination ability between REM and NREM for a subject, as shown in Fig. 6. The SD1 profile (top) was found to have discrimination performance between REM and NREM, but it did not have such performance for another subject. Therefore, the SD1 parameter is marked by high dependence on subjects, with the SD2 parameter being characterized by slight dependence. However, the profile of the SD2/SD1 ratio has characteristics that are independent of subjects during sleep. When an independent parameter is considered, the threshold in the SD2/SD1 ratio between REM and NREM may become 2.0, under the condition that a 2 min window is applied throughout the night. Determination of this threshold can provide sleep information with a ratio higher than 2.0 corresponding to NREM and a lower ratio corresponding to REM to monitor sleep stages in real time. The subject-to-subject variation in the features of SD1 and SD2 parameters should be further investigated to evaluate clinical sleep stages. In addition, all the profiles of SD2 from NREM showed lower values than those from REM, which represents parasympathetic activity over sympathetic activity, but several reversals were observed. The profiles of any Poincare plot parameters for REM and NREM were expected to be linked with sleep efficiency but could not be proven. 

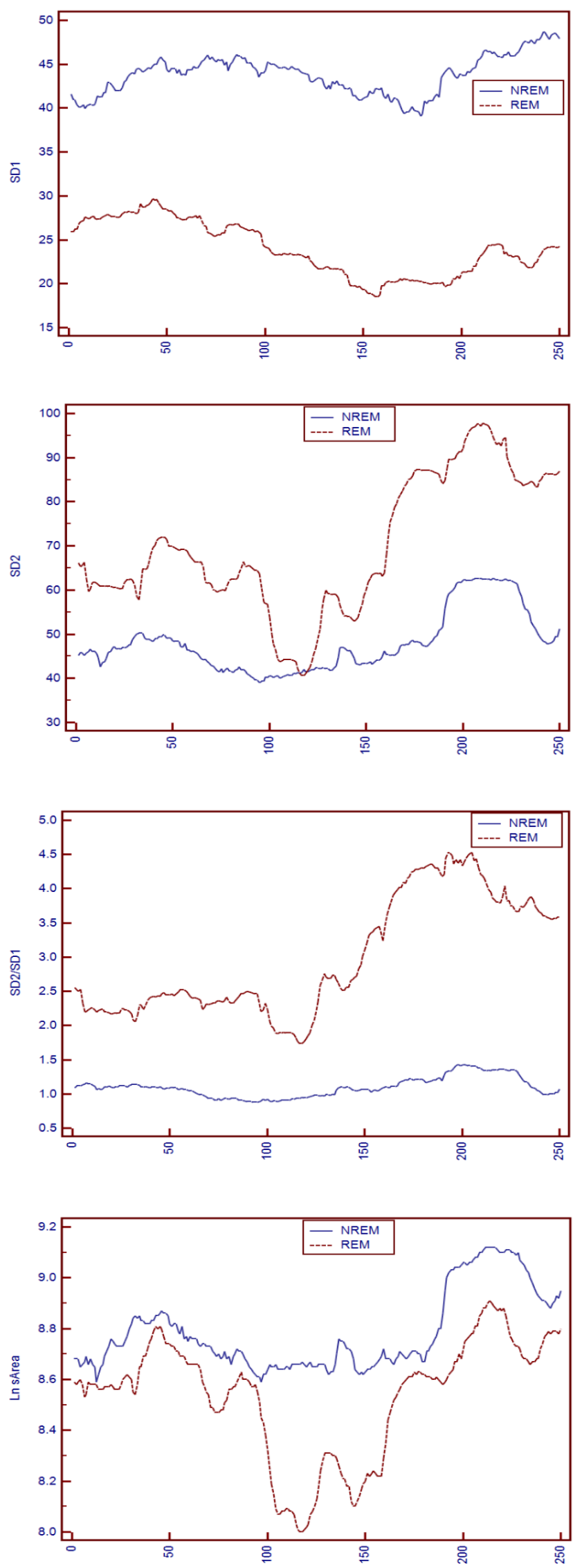

Fig. 6. Profiles of Poincare plot parameters of the 240 results using a 2 min window for a subject.

\section{CONCLUSION}

Four Poincare plot parameters were investigated to distinguish REM and NREM during sleep: SD1, SD2, the SD2/SD1 ratio, and the Ln sArea. A 2 min moving window with a time shift of $2 \mathrm{~s}$ was applied to obtain all the profiles of individual Poincare plot parameters for a $10 \mathrm{~min}$ target HRV segment. The SD2/SD1 ratio was highly associated with the discrimination performance between REM and NREM, followed by the SD of all Ln sAreas and the SD2. A decrease in SD2 from NREM reflects parasympathetic predominance over sympathetic activity. This finding indicates that the SD2 parameter could replace Ln HF obtained by spectral analysis, which corresponds to the parasympathetic tone. Additional findings include that the value of Ln sArea remained constant independent of sleep stages because SD1 and SD2 changed with a reciprocal activation of these parameters in REM and NREM. This result suggests that the degree of consistency of the Ln sArea, mainly due to its association with dynamic ANS activities during sleep, could be a useful predictor for assessing the effectiveness of patient treatment or health status linked to sleep disorders. A new parameter related to the Poincare plot, NREM/REM (\%), was proposed to evaluate the most affected parameters in the two sleep stages. NREM/REM (\%) will be significantly altered in one of the Poincare plot parameters for patients with sleep deprivation compared to healthy individuals. For future sleep HRV studies, Poincare plot parameters in a nonlinear HRV analysis will be investigated to assess a pathological or physiological state throughout the sleep period.

\section{ACKNOWLEDGMENT}

This work was supported by the Hallym University Research Fund (HRF-202008-017).

\section{REFERENCES}

[1] Task Force of the European Society of Cardiology and the North American Society of Pacing and Electrophysiology, "Heart rate variability: standards of measurement, physiological interpretation and clinical use," Circulation. 1995;93(5):1043-1065.

[2] Phyllis K. Stein, Peter P. Domitrovich, Nelson Hui, Pentti Rautaharju, John Gottdiener, "Sometimes higher heart rate variability is not better heart rate variability: results of graphical and nonlinear analyses," Journal of Cardiovascular Electrophysiology. 2005;16(9):954959.

[3] D.E. Vigo, L.N. Siri, D.P. Cardinali, "Heart rate variability: A tool to explore autonomic nervous system activity in health and disease," Psychiatry and Neuroscience Update. 2018:113-126.

[4] J.D. Scheff, B. Griffel, S.A. Corbett, S.E. Calvano, I.P. Androulakis, "On heart rate variability and autonomic activity in homeostasis and in systemic inflammation," Mathematical Biosciences. 2015;252:36-44.

[5] Ateke Goshvarpour, Ataollah Abbasi, Atefeh 
Goshvarpour, "Indices from lagged Poincare plots of heart rate variability: an efficient nonlinear tool for emotion discrimination," Australian Physical \& Engineering Sciences in Medicine. 2017;40(2):277-287.

[6] Atefeh Goshvarpour, Ateke Goshvarpour, "Poincare indices for analyzing meditative heart rate signals," Biomedical Journal. 2015;38(3):229-234.

[7] Che-Hao Hsu, Ming-Ya Tsai, Go-Shine Huang, TsoChou Lin, Kuen-Pao Chen, Shung-Tai Ho, Liang-Yu Shyu, Chi-Yuan Li, "Poincare plot indexes of heart rate variability detect dynamic autonomic modulation during general anesthesia induction," Acta Anaesthesioloqica Taiwanica: official journal of the Taiwan Society of Anesthesioloqists. 2012;50(1):12-18.

[8] Przemyslaw Guzik, Jaroslaw Piskorski, Tomasz Krauze, Raphael Scheider, Karel H. Wesseling, Andrzej Wykretowicz, Henryk Wysocki, "Correlations between the Poincare plot and conventional heart rate variability parameters assessed during paced breathing," The Journal of Physiological Sciences. 2007;57(1):63-71.

[9] Elena Ioana Iconaru, Constantin Ciucurel, "Hand grip strength variability during serial testing as an entropic biomarker of aging: a Poincare plot analysis, $B M C$ Geriatrics, 2020, vol. 13, no. 1, pp. 12.

[10] Chandan K. Karmakar, Ahsan H. Khandoker, Jayavardhana Gubbi, Marimuthu Palaniswami, "Complex correlation measure: a novel descriptor for Poincare plot," Biomedical Engineering Online. 2009;8(17):1-12.

[11] Senthil Packiasabapathy, Varesh Prasad, Valluvan Rangasamy, David Popok, Xinling Xu, Victor Novack, Balachundhar Subramaniam, "Cardiac surgical outcome prediction by blood pressure variability indices Poincare plot and coefficient of variation: a retrospective study, BMC Anesthesiology. 2020;20(1):1-12.

[12] Ernesto Kufoy, Jose-Alberto Palma, Jon Lopez, Manuel Alegre, Elena Urrestarazu, Julio Artieda, Jorge Iriarte, "Changes in the heart rate variability in patients with obstructive sleep apnea and its response to acute CPAP treatment," PLoS One. 2012;7(3):e33769.

[13] Vanessa Cristina Cunha Seueira, Pamela Martin Bandeira, Joao Carlos Moreno Azevedo, "Heart rate variability in adults with obstructive sleep apnea: a systematic review," Sleep Science. 2019;12(3):214-221.

[14] Eleonora Tobaldini, Lino Nobili, Silvia Strada, Karina R. Casali, Alberto Braghiroli, Nicola Montano, "Heart rate variability in normal and pathological sleep," frontiers in Physiology. 2013;4(294):1-11.

[15] Konstantinos Chaidas, Marian Tsaoussoglou, Emmanouel Theodorou, Loukia Lianou, George Chrousos, Athanasios G. Kaditis, "Poincare plot width, morning urine norepinephrine levels, and autonomic imbalance in children with obstructive sleep apnea," Pediatric Neurology. 2014;51(2):246-251.
[16] Brando V, Castro-Zaballa S, Falconi A, Torterolo P, Migliaro ER, "Statistical, spectral and non-linear analysis of the heart rate variability during wakefulness and sleep," Europe PMC. 2014;152(1):32-46.

[17] Brennan, M., Palaniswami, M., and Kamen, P. Do existing measures of Poincare plot geometry reflect nonlinear features of heart rate variability. IEEE Transactions on Biomedical Engineering. 2001;48:1342-1347.

[18] Jeom Keun Kim, Jae Mok Ahn, "New marker for vascular health based on the Poincare plot analysis using acceleration plethysmogram," International Journal of Applied Engineering Research. 2018;13(21):1541715423.

[19] Evgeniya Gospodinova, "Graphical methods for nonlinear analysis of electrocardiographic data," International Conference on Innovations in Science and Education. 2019; doi:10.12955/cbup.v7.1497.

[20] Dong-Kyu Kim, Jae Mok Ahn, "Heart rate variability assessment by the entropy parameters during sleep," Internation Journal of Innovative Technology and Exploring Engineering. 2020;9(9):468-472. 